\title{
Stationary and dispersive features in resonant inelastic soft X-ray scattering at the Ge 3p resonances
}

\author{
C.J. Glover ${ }^{\mathrm{a}}$, T. Schmitt ${ }^{\mathrm{b}}$, M. Mattesini ${ }^{\mathrm{c}}$, M. Adell ${ }^{\mathrm{d}}$, L. Ilver ${ }^{\mathrm{d}}$, J. Kanski ${ }^{\mathrm{d}}$, L. Kjeldgaard ${ }^{\mathrm{e}}$, M. Agåker $^{\mathrm{f}}$, \\ N. Mårtensson ${ }^{e, f}$, R. Ahuja ${ }^{f}$, J. Nordgren ${ }^{f}$, J.-E. Rubensson ${ }^{f, *}$ \\ a Department of Electronic Materials Engineering, Australian National University, Canberra, ACT 0200, Australia \\ b SLS, Paul Scherrer Institut, PSI West, CH-5232 Villigen PSI, Switzerland \\ c Departamento de Física de la Tierra, Astronomía y Astrofísica I, Universidad Complutense de Madrid, E-28040, Spain \\ d Chalmers University of Technology, SE-412 96 Göteborg, Sweden \\ e MAX-lab, Lund University, P.O. Box 118, SE-22100 Lund, Sweden \\ f Department of Physics and Materials Science, Uppsala University, PO Box 530, SE-751 21 Uppsala, Sweden
}

\section{A R T I C L E I N F O}

\section{Article history:}

Received 3 February 2009

Received in revised form 25 May 2009

Accepted 27 May 2009

Available online 6 June 2009

\section{Keywords:}

Soft X-ray scattering (RIXS)

Ultrafast dynamics

Semiconductors

Spectroscopy

Synchrotron radiation

\begin{abstract}
A B S T R A C T
Resonant inelastic soft X-ray scattering at the $3 p$ resonances in crystalline Ge is presented. Both stationary and dispersive features are observed in a wide energy range above as well as below the ionization limits. These observations are in agreement with theoretical predictions based on a two-step model where the initially excited electron has no influence on the emission step. Excess population of states in the conduction band is found, and discussed in terms of attosecond electron dynamics.
\end{abstract}

(c) 2009 Elsevier B.V. All rights reserved.

\section{Introduction}

Resonant inelastic soft X-ray scattering (RIXS) has attracted considerable interest as a tool to study electronic structure in solids, atoms and molecules [1,2]. Especially important is the access to dynamics on the ultrafast time scale, set by the lifetime of the intermediate state, and the detuning energy [3]. Rates for various processes are compared to the core hole decay rate in the so-called 'core-hole clock' [4] scheme, and dynamic information is also inherent in interference effects. Here we present a related approach to dynamics in broad-band semiconductors, by considering the scattering process in Fig. 1. In the first step the incoming energy, $\omega_{1}$, is used to excite a core-hole state with energy $\omega_{n l j}$, and an electron with energy $\omega$ in the conduction band. When the lifetime of this state is short, both the latter energies are illdefined and features in the corresponding absorption spectrum are smeared out. As a photon with energy $\omega_{2}$ is emitted, energy conservation determines $\omega_{\text {exc }}$, and if $\omega_{n^{\prime} l^{\prime} j^{\prime}}$ corresponds to a welldefined long-lived state, also the electron energy, $\omega$, becomes well

\footnotetext{
* Corresponding author. Tel.: +46184713562.

E-mail address: jan-erik.rubensson@fysik.uu.se (J.-E. Rubensson).
}

defined. In this sense, the second step of the scattering process can be regarded as a snap-shot, monitoring the energy of the excited electron after the lifetime of the intermediate state. Here we present RIXS spectra of Ge excited at short-lived (around 200 as) intermediate states with $3 \mathrm{p}_{3 / 2,1 / 2}$ vacancies. Long-lived (around $4 \mathrm{fs}$ ) final states with $3 \mathrm{~d}_{5 / 2,3 / 2}$ vacancies are populated, and the spectra demonstrate that the electron dynamically populates certain states in the conduction band. In addition, we show that, contrary to what is often stated, localization/delocalization of the excited electron is not essential for the dispersion properties of RIXS features.

\section{Experimental}

The measurements were made at beamline I511 [5] at MAXlab, Sweden. RIXS spectra of a nominally undoped Ge $\left(\begin{array}{lll}1 & 0 & 0\end{array}\right)$ wafer were measured using a grazing incidence Rowland spectrometer [6] collecting in the direction of polarization of the incoming radiation. The energies of the monochromator and the spectrometer were matched using the diffuse reflection of the incoming radiation, and calibrated to better than $0.1 \mathrm{eV}$. Diffuse reflection also established the spectrometer function (full width at half maximum $(\mathrm{FWHM}) \approx 0.3 \mathrm{eV}$ at $95 \mathrm{eV})$. The resolution of the monochromator 
(a)

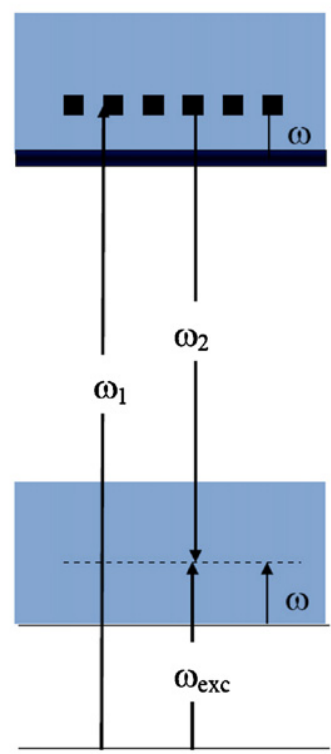

(b)

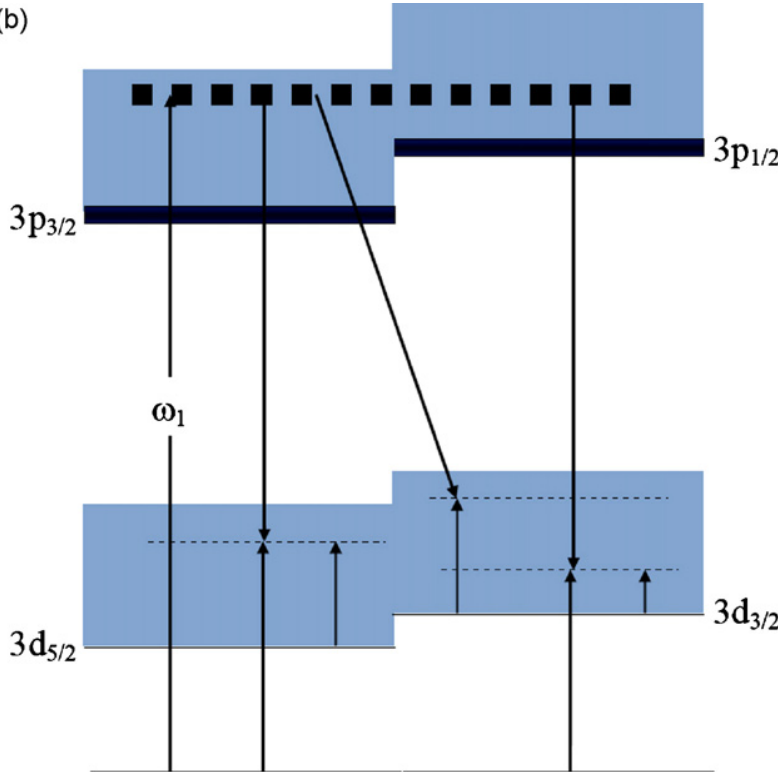

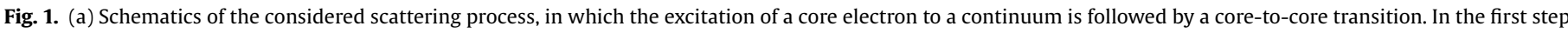

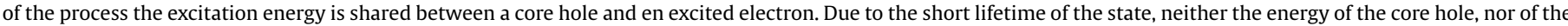

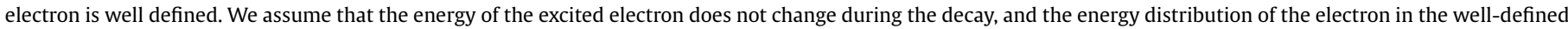

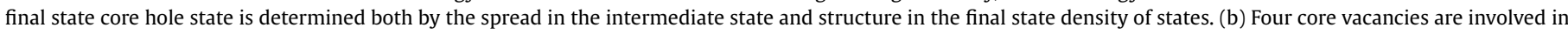

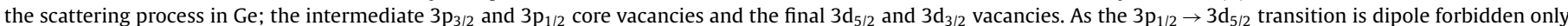
three different scattering paths contribute to the spectra, each corresponding to a well-defined electron energy.

was set to $0.05 \mathrm{eV}$ at $125 \mathrm{eV}$, allowing $\sim 10^{12}$ photons/s to be delivered in a $20 \mu \mathrm{m} \times 50 \mu \mathrm{m}$ spot. Spectra were acquired for $\sim 0.5 \mathrm{~h}$ each.

\section{Results and discussion}

The measured RIXS spectra of crystalline Ge excited around the $3 p$ thresholds are compared to theoretical predictions in Fig. 2. Well above threshold, the spectra are dominated by two broad, excitation-energy independent structures peaking at $92.1 \mathrm{eV}$ and $95.9 \mathrm{eV}$. In addition, much narrower features, dispersing linearly with the excitation energy, appear throughout the excitationenergy range (120-135 eV). The theoretical foundation for RIXS was thoroughly discussed several years ago [7,8], and the description is still being refined [9]. In Fig. 2 the theory curves have been constructed from the scattering theory for the cross-section formulated by Tulkki and Åberg [8]:

$$
\begin{aligned}
\frac{d \sigma\left(\omega_{1}\right)}{d \omega_{2}} \propto \sum_{j, j^{\prime}} & {\left[\int_{0}^{\infty}\left(\frac{\omega_{2}}{\omega_{1}}\right) \frac{\left(\omega_{3 \mathrm{p}_{j}}-\omega_{3 \mathrm{~d}_{j^{\prime}}}\right) g_{3 \mathrm{~d}_{j^{\prime}} \mathrm{p}_{j}}\left(\omega_{3 \mathrm{p}_{j}}+\omega\right)\left(d g_{3 \mathrm{p}_{j}} / d \omega\right)}{\left(\omega_{3 \mathrm{p}_{j}}+\omega-\omega_{1}\right)^{2}+\Gamma_{3 \mathrm{p}_{j}}^{2}}\right.} \\
& \left.\times \delta\left(\omega_{1}-\omega_{3 \mathrm{~d}_{j^{\prime}}}-\omega-\omega_{2}\right) d \omega\right]
\end{aligned}
$$

where $\Gamma_{3 \mathrm{p}_{j}}$ is the lifetime width of the intermediate state, $g_{3 \mathrm{~d}_{j^{\prime}}, 3 \mathrm{p}_{j}}$ is the oscillator strength for transitions between $3 \mathrm{p}_{j}$ and $3 \mathrm{~d}_{j^{\prime}}$ hole levels with binding energies $\omega_{3 \mathrm{p}_{j}}$ and $\omega_{3 \mathrm{~d}_{j^{\prime}}}$, respectively, and $d g_{3 \mathrm{p}_{j}} / d \omega$ is the oscillator strength per energy for exciting an electron from the $3 \mathrm{p}_{j}$ level to an energy $\omega$ in the conduction band. The predictions use $d g_{3 \mathrm{p}_{j}} / d \omega$, based on band structure calculations, performed for cubic germanium (Fd3m, $a=5.6574 \AA$ ) by using the full-potential linearized augmented plane wave method (FP-LAPW) as implemented in the WIEN2k package [10]. Exchange and correlation effects were taken into account through the local-density approximation (LDA) as parameterized by Perdew and Wang [11]. The influence of the 3p core hole on the electronic band states were included by formally treating the excited atom as an impurity in a supercell of dimension $2 \times 2 \times 2$. The $d g_{3 \mathrm{p}_{j}} / d \omega$ was then calculated by multiplying the density of states by a dipole matrix element that accounts for the transitions between the $l$ core states to the $l \pm 1$ components of the conduction band.

The conduction band density of states (DOS) is typical for the diamond-structured elemental 4th period semiconductors (Fig. 3). The introduction of a $3 p$ core hole has little impact on the DOS, the Coulomb potential basically uniformly shifting the features to lower energies, without evidence of localization. On the other hand, the matrix elements strongly emphasize s-type DOS, so that $d g_{3 \mathrm{p}_{j}} / d \omega$ is dominated by a fairly sharp structure close to the bottom of the conduction band.

The agreement between the experimental results and the predictions in Fig. 2 is excellent. Especially, both stationary features, with a width corresponding to the lifetime broadening of the intermediate states, and a set of narrow dispersive features, are reproduced in the model. The discrepancy, beginning in the $124.5 \mathrm{eV}$ excited spectrum is due to the emergence of a second dispersing feature not predicted by the model. Before further discussing this feature we make some general comments on the dispersion behavior.

To arrive at Eq. (1) the intermediate and final states are assumed to be orthogonal, and since interference is neglected, a two-step picture can be maintained. In the first step the electron is excited according to $d g_{3 \mathrm{p}_{j}} / d \omega$, with an uncertainty in $\omega$ due to the finite lifetime of the $3 p$ states (see Fig. 1), described by a Lorentz function centered at $\omega_{0}=\omega_{1}-\omega_{3 p_{j}}$, set by the denominator. The second emission step to well-defined $3 \mathrm{~d}$ hole states then unambiguously determines $\omega$ via $\delta\left(\omega_{1}-\omega_{3 \mathrm{~d}_{j^{\prime}}}-\omega-\omega_{2}\right)$. Thus, the decay can be said to map the energy distribution of the electron in the intermedi- 


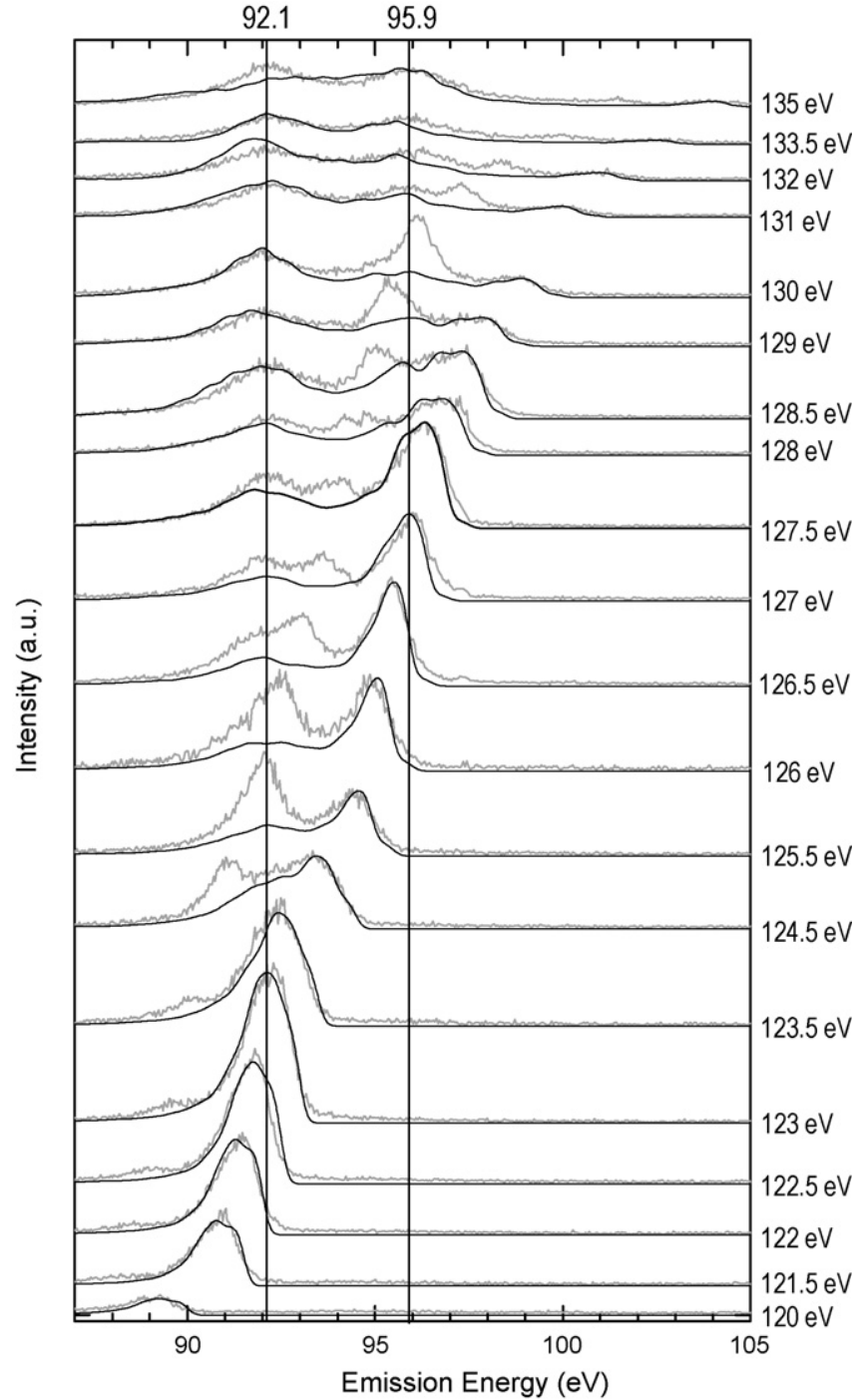

Fig. 2. Experimental and theoretical RIXS spectra on the emission energy scale, as a function of excitation energy (shown on the right). The theoretical curves have been normalized to main peak height for comparison of the spectral shape. They are based on Eq. (1), and the theoretical oscillator strength, $d g_{3 \mathrm{p}_{\mathrm{j}}} / d \omega$, shown in Fig. 3, with the statistical intensity ratio $\left(d g_{3 \mathrm{p}_{3 / 2}} / d \omega\right) /\left(d g_{3 \mathrm{p}_{1 / 2}} / d \omega\right)=2$. We use $\omega_{3 \mathrm{p}_{3 / 2}}=122.1 \mathrm{eV}$, $\omega_{3 \mathrm{p}_{1 / 2}}=126.5 \mathrm{eV}, \omega_{3 \mathrm{~d}_{5 / 2}}=30 \mathrm{eV}, \omega_{3 \mathrm{~d}_{3 / 2}}=30.6 \mathrm{eV}$, and $\Gamma_{3 \mathrm{p}_{j}}=2.1 \mathrm{eV}$, all close to literature values [18]. The final state lifetime width is accounted for by replacement of the $\delta$-function by a Lorentzian with $\Gamma_{3 \mathrm{p}_{i}}=0.3 \mathrm{eV}$, and for the total spectral width we convolute with a Gaussian (FWHM $=0.42 \mathrm{eV}$ ) function, which takes both inherent broadening ( $\mathrm{FWHM}=0.3 \mathrm{eV}$ ) [18] and the spectrometer function ( $\mathrm{FWHM}=0.3 \mathrm{eV}$ ) into account. The finite width of the monochromator function has only a minute influence on the predictions. To improve the agreement we use the core-to-core oscillator strength ratios $g_{3 \mathrm{~d}_{5 / 2}, 3 \mathrm{p}_{3 / 2}} / g_{3 \mathrm{~d}_{3 / 2}, 3 \mathrm{p}_{3 / 2}} / g_{3 \mathrm{~d}_{3 / 2}, 3 \mathrm{p}_{1 / 2}}=6 / 3 / 4.5$, which differ slightly from the statistical weights: $6 / 5 / 3$. See text for a discussion of the mechanisms for peak formation.

ate state. Essentially, this is determined by the product of $d g_{3 \mathrm{p}_{j}} / d \omega$ and the Lorentz function, and thus, the excitation-energy dependence is entirely due to their relative energy position. Stationary as well as dispersive features are now predicted, as can be realized by e.g. considering high above-threshold excitation. Here the Lorentz function is centered at electron energies where $d g_{3 \mathrm{p}_{j}} / d \omega$ is almost constant, and therefore the emission spectrum here takes the form of the Lorentz function, with the center at the constant emission energy $\omega_{2}=\omega_{1}-\omega_{3 \mathrm{~d}_{j^{\prime}}}-\omega_{0}=-\omega_{3 \mathrm{~d}_{j^{\prime}}}+\omega_{3 \mathrm{p}_{j}}$, simulating so-called 'normal emission'. Simultaneously, a tail of the Lorentz function
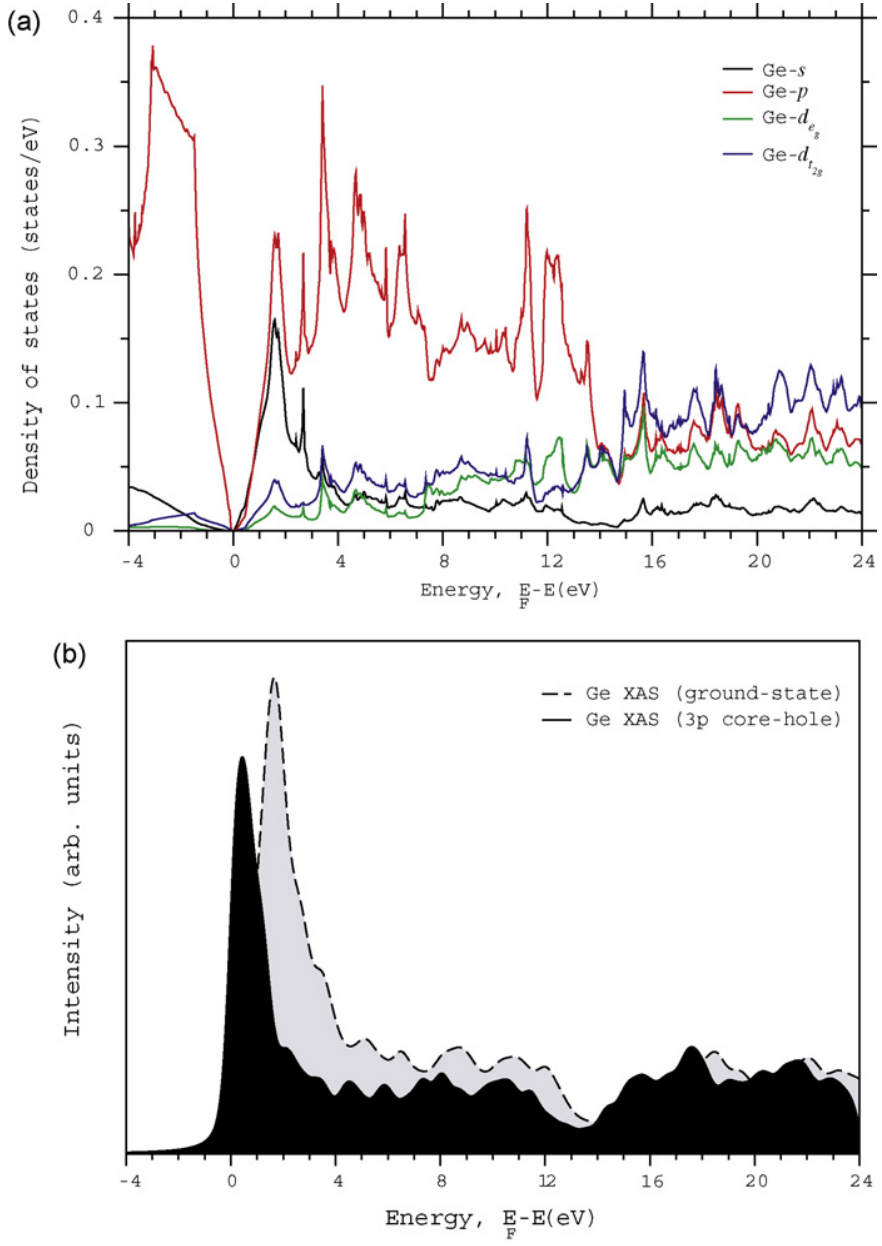

Fig. 3. Calculated s-, p- and d-local partial DOS in the ground state of Ge (a). Matrix elements are calculated for the ground state and in the presence of a $3 p$ core hole to arrive at $d g_{3 \mathrm{p}_{j}} / d \omega$ (b), which is used for the theoretical simulations in Figs. 2 and 4.

extends to low $\omega$ where structures in $d g_{3 \mathrm{p}_{j}} / d \omega$ indeed are sharp enough to determine the form of the emission spectrum (here the Lorentz function is almost flat). As the features in $d g_{3 \mathrm{p}_{j}} / d \omega$ appear at fixed $\omega$ the corresponding spectral features disperse with excitation energy.

'Normal emission' is often associated with an 'incoherent' process, in which the emission has forgotten about how the core hole was excited. Indeed, the scattering spectrum looks similar to emission spectra excited by less selective means such as electrons or ions. Considering the formalism, above, however, it must be emphasized that this similarity is crucially dependent on the distribution of the electron energy, $\omega$ : in Eq. (1) 'normal emission' is retrieved above threshold only because $d g_{3 p_{j}} / d \omega$ lacks structure.

Sharp dispersing features, on the other hand, have often been seen as an indication of localization, and indeed this was also done in an early study of the $3 p$ edge in Ge, in which dispersing features were observed in the electronic decay channel [12]. For continuum excitations linear dispersion of the leading edge of the spectra has been confirmed at below-threshold excitation $[13,14]$, and in the hard X-ray range it has been demonstrated that coreto-core RIXS spectra excited below continuum thresholds can be used to construct 'lifetime-broadening-removed absorption spectra' $[15,16]$. The present results demonstrate that narrow, linearly 


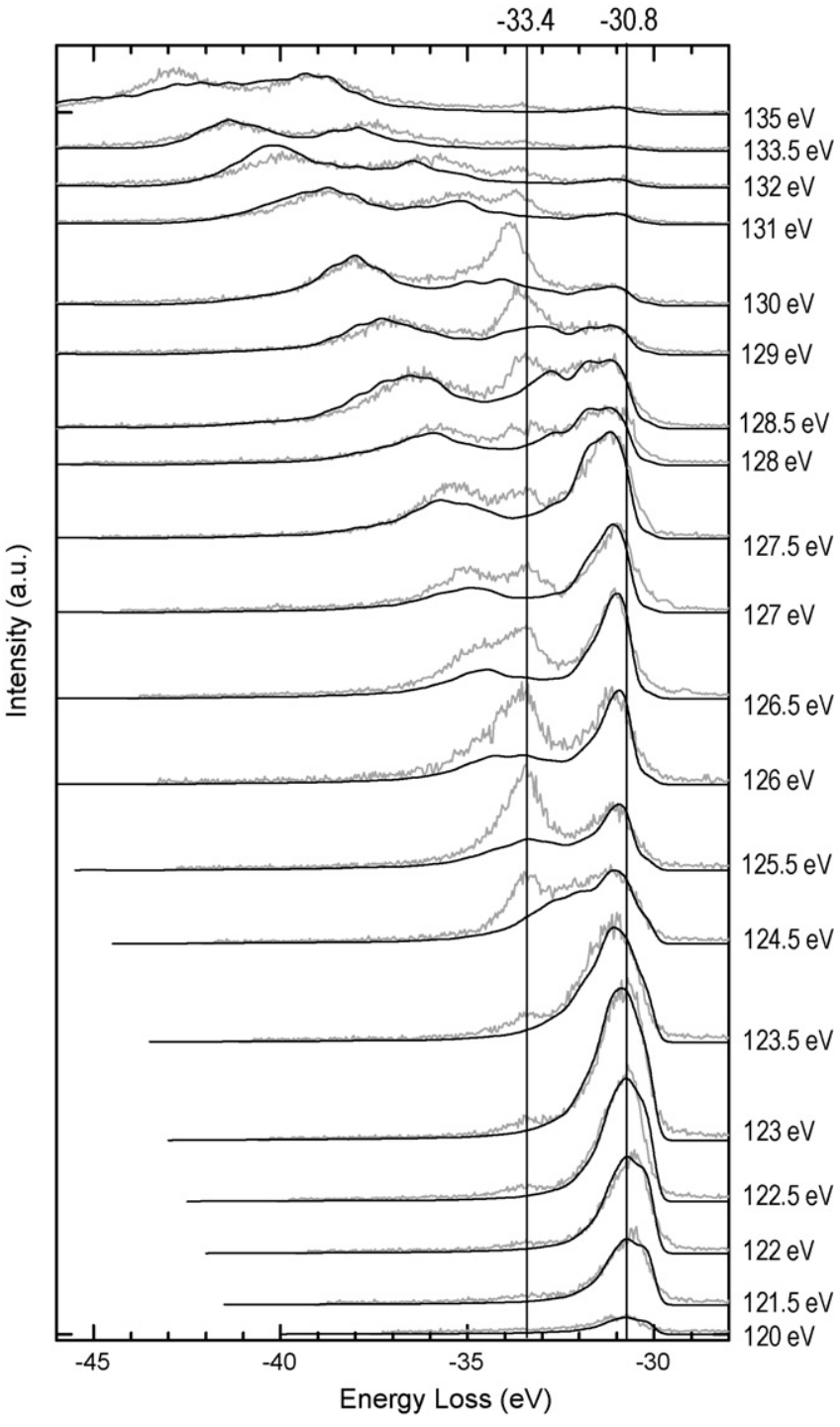

Fig. 4. Scattering data (see caption of Fig. 2) shown on the final state energy (energy loss) scale. The energies of the most prominent features are marked. Note that final states include both $3 \mathrm{~d}_{5 / 2}$ and $3 \mathrm{~d}_{3 / 2}$ vacancies (see Fig. 1 ), separated by $0.6 \mathrm{eV}$. At lower excitation energies (around $122.5 \mathrm{eV}$ ) close to the $3 \mathrm{p}_{3 / 2}$ resonance transitions to $3 \mathrm{~d}_{5 / 2}$ vacancies dominate, giving the highest intensity at $30.8 \mathrm{eV}$ energy loss. At $4.4 \mathrm{eV}$ higher excitation energy the $3 \mathrm{p}_{1 / 2}$ resonance give intensity only to $3 \mathrm{~d}_{3 / 2}$ final states, and consequently the peak shifts by $0.6 \mathrm{eV}$ towards larger energy losses. The resonant peak which is not accounted for by theory goes through two resonances at around $126 \mathrm{eV}$ and $130 \mathrm{eV}$, the excitation energies at which the peak position is $33.4 \mathrm{eV}$ and $34.0 \mathrm{eV}$, respectively. This indicates that, analogously, the shift is due to a change of the spin-orbit coupling of the core vacancy, and that the excited electron is excited to the same energy.

dispersing features can be observed in general for continuum excitations throughout the resonance region, far above and far below thresholds. Sharp dispersing features above continuum thresholds have been presented earlier in GaP [17], but without detailed analysis.

The orthogonality condition used to derive Eq. (1) translates to the assumption that the excited electron does not interact with the rest of the system. It may (or may not) delocalize and/or scatter against electrons and phonons during the absorption-emission process. Since the electron does not influence the decay step, it is not necessarily the electron energy which is measured in the RIXS spectra, but the distribution of any non-interacting 'excess energy' In this sense Eq. (1) is independent of the 'coherency' of the process. This is entirely different in the case where momentum conservation in the scattering process is used to gain information about crystal momenta, where dynamic change of the electron momenta due to electron scattering immediately influences the RIXS spectra [18]. Below we discuss other ways in which Eq. (1) may be violated.

The emission data and the simulation are shown again on the final state energy scale in Fig. 4. The peak(s) centered at energy loss $\sim 30.8 \mathrm{eV}$ are well reproduced by the model. However, the two rather sharp peaks with $0.6 \mathrm{eV}$ splitting at energy loss centered at $33-34 \mathrm{eV}$ are not predicted within the simple theory. Features at $33.4 \mathrm{eV}$ and $34.0 \mathrm{eV}$ are resonantly enhanced at $126 \mathrm{eV}$ and $130 \mathrm{eV}$ excitation energy, respectively. This resonant behavior indicates that they must be associated with the electrons at the same $\omega$, coupled to $3 d_{5 / 2}$ and $3 d_{3 / 2}$ holes, the first being emphasized at the $3 \mathrm{p}_{3 / 2}$ resonance (around $126 \mathrm{eV}$ ), and the latter at $3 \mathrm{p}_{1 / 2}$ (around $130 \mathrm{eV}$ ), in agreement with dipole selection rules. The peaks are situated $2.6 \mathrm{eV}$ above the main (predicted peaks), and they are resonantly enhanced about the same energy distance above the main resonances. In Fig. 3 we see that $\omega$ for the resonance is close to a sharp peak in the local DOS at around $3.5 \mathrm{eV}$ above threshold, $1.8 \mathrm{eV}$ above the main peak. Here $d g_{3 \mathrm{p}_{j}} / d \omega$ is relatively small because the states are dominated by $\mathrm{p}$ and $\mathrm{d}$ character. The p states are dipole forbidden and the matrix elements only give little weight to the d-states, and therefore our theory does not predict the states to be appreciably populated in the RIXS process.

This observation shows the limits of the independent-particle model, and we tentatively assign additional intensity to excess population of these states associated with a rather flat band along which give a large contribution to the density of states, but is dominated by dipole forbidden angular momentum symmetry (see Fig. 5). These states may be populated due to the dynamics in the scattering process. Two mechanisms may be envisioned: any symmetry-breaking coupling involving the excited electron and the local electronic system allows for a dynamic rearrangement during the lifetime of the intermediate state (around 200 as) to populate the states which primarily have p- and d-character. Another possibility is that the relatively local nature of these states gives them additional intensity in the scattering process. An electron wavepacket created in a first step does not have time to recede far from the site during the duration of the scattering process. Given sufficient Coulomb interaction this may give excess intensity to localized final states. Note that a two-step picture where an exciton is assumed to form already in the first step is unphysical, because the energy uncertainty in the $3 p$ states is too large, or equivalently the time is too short for the formation of an exciton. This type of dynamic behaviour assumes an influence of the excited electron on the second step of the process, which cannot be described by Eq. (1). Such dynamics also puts a limit to the applicability of such equations for constructing 'lifetime-broadening-removed absorption spectra' $[15,16]$.

Finally, we relate the present results to the observation of a sharp peak at around $4 \mathrm{eV}$ energy loss in L-edge RIXS of $\mathrm{Si}$, when excited below the edge $[18,19]$. Predictions based on band structure calculations seem unable to explain this feature [18] and it has been assigned to a surprisingly prominent exciton state [19]. Here the detuning energy implies a shortening of the scattering duration time to below femtoseconds, and we speculate that also in this case the time scale become essential for the final state population. Together with the present Ge results, this suggests that localization in simple semiconductors sets an upper time constraint for electron/hole mobility. 


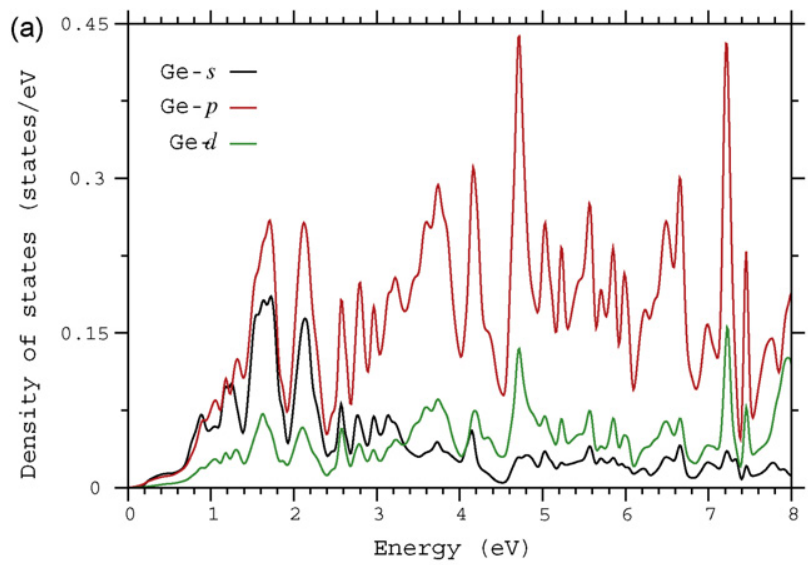

(b)

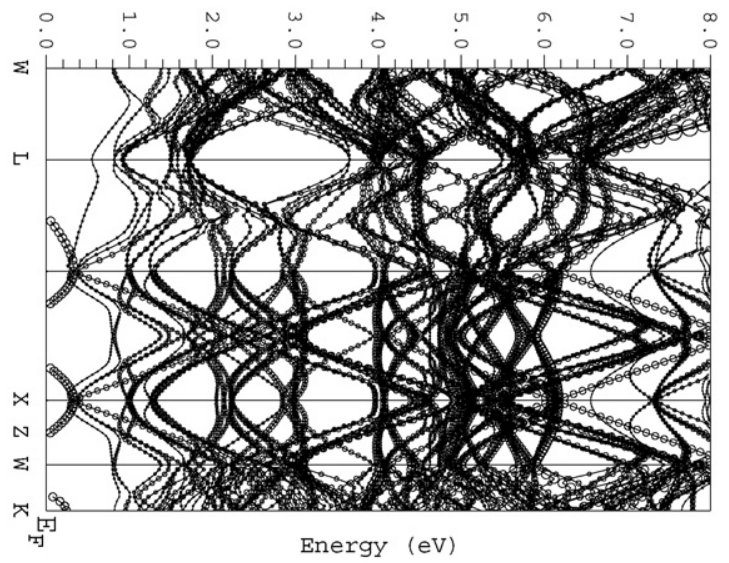

(c)

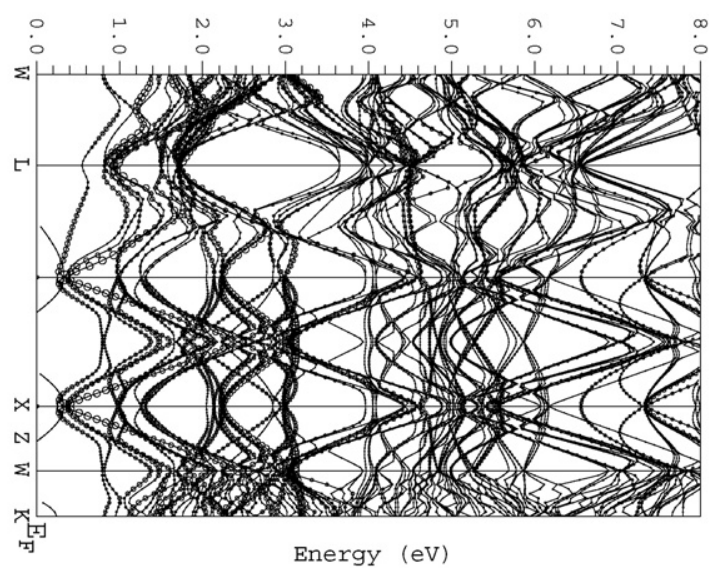

(d)

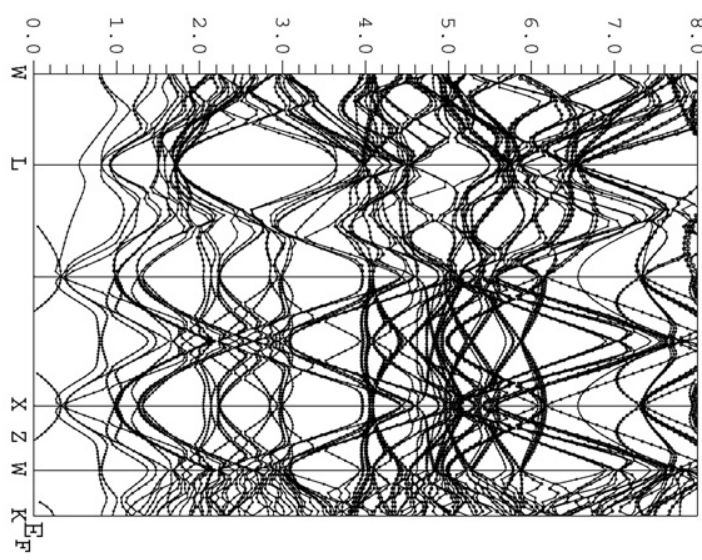

Fig. 5. The s-, p-, and d-density of states in the presence of a core hole (a). In (b), (c), and (d) we show the band-character plots, where the radii of the circles are proportional to $\mathrm{p}-, \mathrm{s}$ - and $\mathrm{d}$-character of the states. It is seen that a fairly flat band around $4 \mathrm{eV}$ is dominated by $\mathrm{p}$-character.

\section{Conclusions}

In summary, we have demonstrated that both stationary 'normal emission' and sharp dispersing features in RIXS can be described in a two-step picture where there is no interaction between the excited electron and the rest of the electron system. The fact that localization is not prerequisite substantially extends the applicability of RIXS, and it will be of importance at the new generation VUV-FEL sources, where high-precision measurements will be performed. Furthermore, we have demonstrated that, albeit a good overall agreement, the simple model does not account for all details. Dynamics during the scattering process can be addressed: we find excess population of localized states in the conduction band during the fast time scale of the scattering process.

\section{Acknowledgements}

The authors gratefully acknowledge the support from the Australian Research Council, Swedish Research Council, the Spanish Ministry of Education and Science (Grant No. CGL2008-00891), the Swedish Foundation for Strategic Research, and the Göran Gustafsson Foundation.

\section{References}

[1] See various articles in the topical issue of J. Electron Spectrosc. (2000) 110-111.
[2] A. Kotani, S. Shin, Rev. Mod. Phys. 73 (2001) 203.

[3] F. Gel'mukhanov, P. Sałek, T. Privalov, H. Ågren, Phys. Rev. A 59 (1999) 380.

[4] A. Föhlisch, P. Feulner, F. Hennies, A. Fink, D. Menzel, D. Sanchez-Portal, P.M. Echenique, W. Wurth, Nature 436 (2005) 373, and Refs. therein.

[5] R. Denecke, P. Väterlein, M. Bässler, N. Wassdahl, S. Butorin, A. Nilsson, J.-E. Rubensson, N. Mårtensson, J. Nordgren, R. Nyholm, J. Electron Spectrosc. 105 (1999) 971.

[6] J. Nordgren, G. Bray, S. Cramm, R. Nyholm, J.-E. Rubensson, N. Wassdahl, Rev. Sci. Instrum. 60 (1989) 1690.

[7] T. Åberg, J. Tulkki, in: B. Crasemann (Ed.), Atomic Inner-shell Physics, Plenum Press, New York, 1985, p. 419.

[8] J. Tulkki, T. Åberg, J. Phys. B 15 (1982) L435.

[9] T. Fujikawa, T. Konishi, T. Fukamachi, J. Electron Spectrosc. 134 (2004) 195.

[10] P. Blaha, K. Schwarz, G.K.H. Madsen, D. Kvasnicka, J. Luitz, WIEN2K, An Augmented Plane Wave Plus Local Orbitals Program for Calculating Crystal Properties, Vienna University of Technology, Austria, 2001.

[11] J.P. Perdew, Y. Wang, Phys. Rev. B 45 (1992) 13244.

[12] A. Kivimäki, H. Aksela, S. Aksela, O.-P. Sairanen, Phys. Rev. B 47 (1993) 4181.

[13] J.-E. Rubensson, J. Electron Spectrosc. 110-111 (2000) 135.

[14] M. Magnuson, J.-E. Rubensson, A. Föhlisch, N. Wassdahl, A. Nilsson, N. Mårtensson, Phys. Rev. B68 (2003) 45119.

[15] H. Hayashi, R. Takeda, Y. Udagawa, T. Nakamura, H. Miyagawa, H. Shoji, S. Nanao, N. Kawamura, Phys. Rev. B 68 (2003) 045122.

[16] H. Hayashi, R. Takeda, M. Kawata, Y. Udagawa, N. Kawamura, Y. Watanabe, S. Nanao, Phys. Rev. B 70 (2004) 155113.

[17] L. Lin, G.T. Woods, T.A. Callcott, Phys. Rev. B 63 (2001) 235107.

[18] S. Eisebitt, J. Lüning, J.-E. Rubensson, A. Settels, P.H. Dederichs, W. Eberhardt, S.N. Patitsas, T. Tiedje, J. Electron Spectrosc. Relat. Phenom. 93 (1998) 245.

[19] S. Shin, A. Agui, M. Watanabe, M. Fujisawa, Y. Tezuka, T. Ishii, Phys. Rev. B 53 (1996) 15660. 